\title{
Miliary tuberculosis after in vitro fertilization and embryo transplantation
}

\author{
Hongbo Liu, Li Zhao
}

Department of Respiratory Medicine, Shengjing Hospital of China Medical University, Shenyang, Liaoning China. 110004

\begin{abstract}
Background: With the development of assisted reproductive technology, more patients with infertility prefer to get pregnant by in vitro fertilization and embryo transplantation (IVF-ET). But the indications of IVF-ET must be strictly controlled by the clinicians.

Case report: We described a case of a 29-year-old pregnant Chinese woman with military tuberculosis. She was admitted to the hospital with complaints of fever and shortness of breath. She received IVF-ET 83 days ago, because of infertility with bilateraltubal occlusion, and was in pregnancy status. We presented the clinical, laboratory and two chest CT scan features of the patient. The second chest CT scan showed randomly distributed, innumerable tiny, military nodules throughout the lungs. The fluorescence quantitative PCR of the bronchoalveolar lavage fluid (BALF) was positive for Mycobacterium tuberculosis. The M. tuberculosis was cultured from BALF on Day 29. So we made the diagnosis of military tuberculosis.

Conclusion: It is important to rule out tuberculosis for the infertility patients especially with bilateraltubal occlusion before IVF-ET in order to avoid tuberculosis disseminate during pregnancy.
\end{abstract}

Key words: military tuberculosis; in vitro fertilization and embryo transplantation.

DOI: http://dx.doi.org/10.4314/ahs.v15i2.50

\section{Introduction}

Tuberculosis has been a major health problem all over the world. Currently, about one-third of the human population is infected with tuberculosis worldwide ${ }^{1}$. Genital tuberculosis is one of the most common causes of female infertility ${ }^{2}$.With the development of assisted reproductive technology, more infertility patients prefer to get pregnant by in vitro fertilization and embryo transplantation (IVF-ET). But we found one patient who showed tuberculosis dissemination during pregnancy after IVF-ET.

\section{Case report}

A 29-year-old woman was admitted to the hospital with complaints of fever and shortness of breath. She got

\section{Corresponding author:}

Hongbo Liu

Department of Respiratory Medicine, Shengjing Hospital of China Medical University, Shenyang, Liaoning China. 110004 E-mail:18940254125@189.cn a fever for five days with no reasons and felt breathlesson exertion for two days. The highest temperature was $38.4^{\circ} \mathrm{C}$, she had no chills and rogors. She denied any complaints of cough, sputum, abdominal pain, diarrhea, urinary frequency, urgency of urination, urodynia, night sweats or weight loss. She had received amoxicillin but without any effect. Her remaining medical history was bilateral tubal occlusion. She had amenorrhea for three and a half month, and had received IVF-ET 83 days ago. She was in pregnancy status for 69 days according to the high level of the human chorionic gonadotropin (HCG) in blood, and two gestational sac and embryocardia had been found in her uterus through the ultrasonography 55 days ago. After IVF-ET she had taken $10 \mathrm{mg}$ dydrogesterone orally and $10 \mathrm{mg}$ progesterone by intramuscular injection per day for one month. She had no history of prior pulmonary infection.

On physical examination, her temperature was $37.9^{\circ} \mathrm{C}$; $\mathrm{BP}, 117 / 79 \mathrm{mmHg}$, pulse, 110 beats/min, respiratory frequency, 20 breaths/min and oxygen saturation, $96 \%$ on room air. Head and neck examination results were normal without nuchal rigidity. Chest examination was normal. Cardiac examination revealed a regular rhythm, no gallop or murmur. Abdomen was not tender, with 
normal bowel sounds without hepatosplenomegaly. liver function tests were normal. ESR was $32 \mathrm{~mm} / \mathrm{h}$. - Rapid HIV screening was negative. ECG showed sinus evidence of any skin rash. tachycardia. Chest CT scan (Fig 1) showed consolidation and effusion in the middle lobe of the right lung and hyperplasia and calcified lesions in the bilateral upwere 5570 cells $/ \mu \mathrm{L}$ with $80.4 \%$ neutrophils, hemoglo- per lobes of the lung. According to these results the bin level was $10.4 \mathrm{~g} / \mathrm{dL}$ and platelet count was 315,000 cell $/ \mu \mathrm{L}$. CRP was $18.2 \mathrm{mg} / \mathrm{l}$. Electrolytes, renal and tuberculosis diagnosis was made.

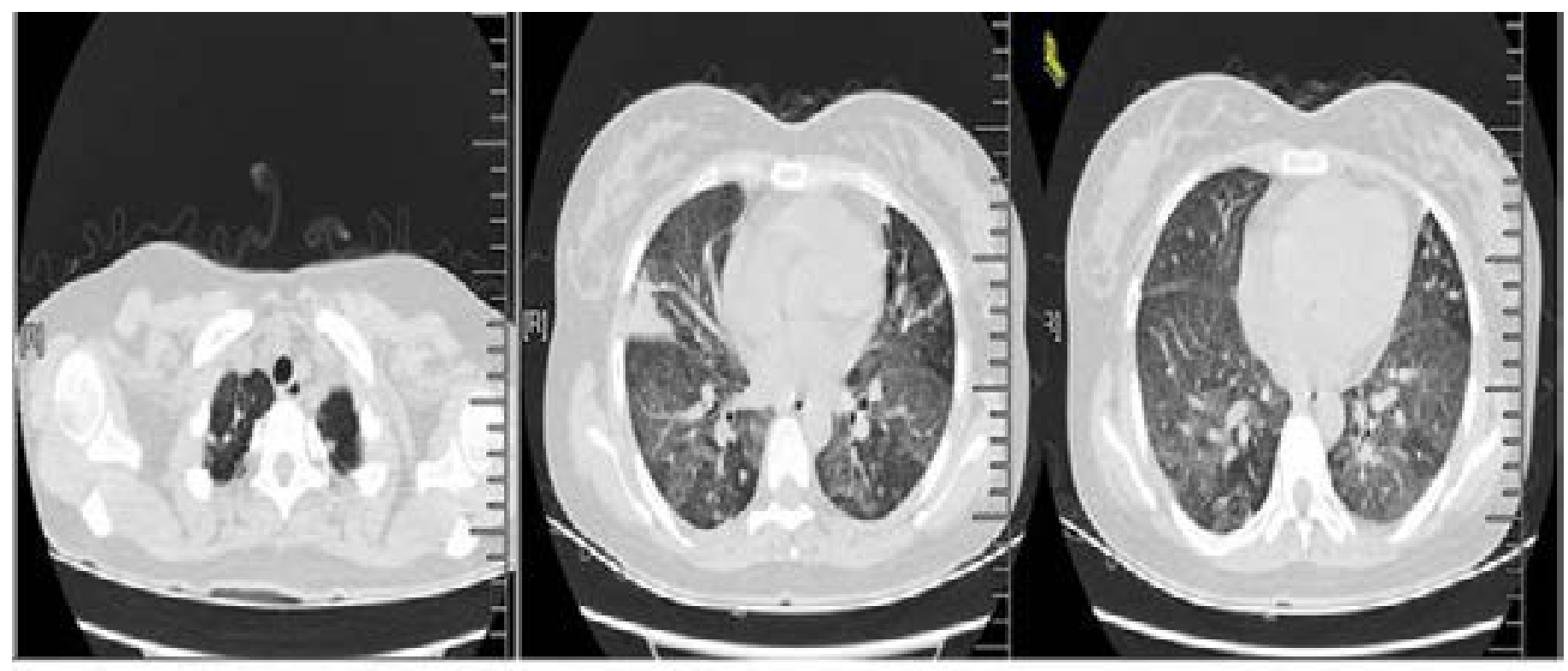

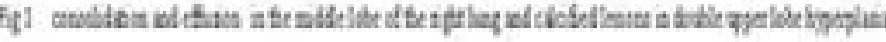

The patient was treated with Azithromycin and Ce- throughout the lungs. The branchofiberoscope test was furoxime. However, she developed severe shortness of performed. The bronchoalveolar lavage fluid (BALF) breath and the temperature reaching $40^{\circ} \mathrm{C}$, respiratory did not find acid-fast bacilli. But the fluorescence quanfrequency 26 breaths/min, oxygen saturation $90 \%$ in titative PCR of BALF was positive for Mycobacterium room air. Arterial blood gas analysis revealed PH 7.43, tuberculosis and M. tuberculosis was cultured on Day $\mathrm{PaO}^{2} 59.3 \mathrm{mmHg}$ and $\mathrm{PaCO}^{2} 32 \mathrm{mmHg}$. She got serious. 29 from BALF. Thus, the diagnosis was revised to MilFive days later, the other chest CT scan (Fig 2) showed itary tuberculosis.

randomly distributed, innumerable tiny, military nodules

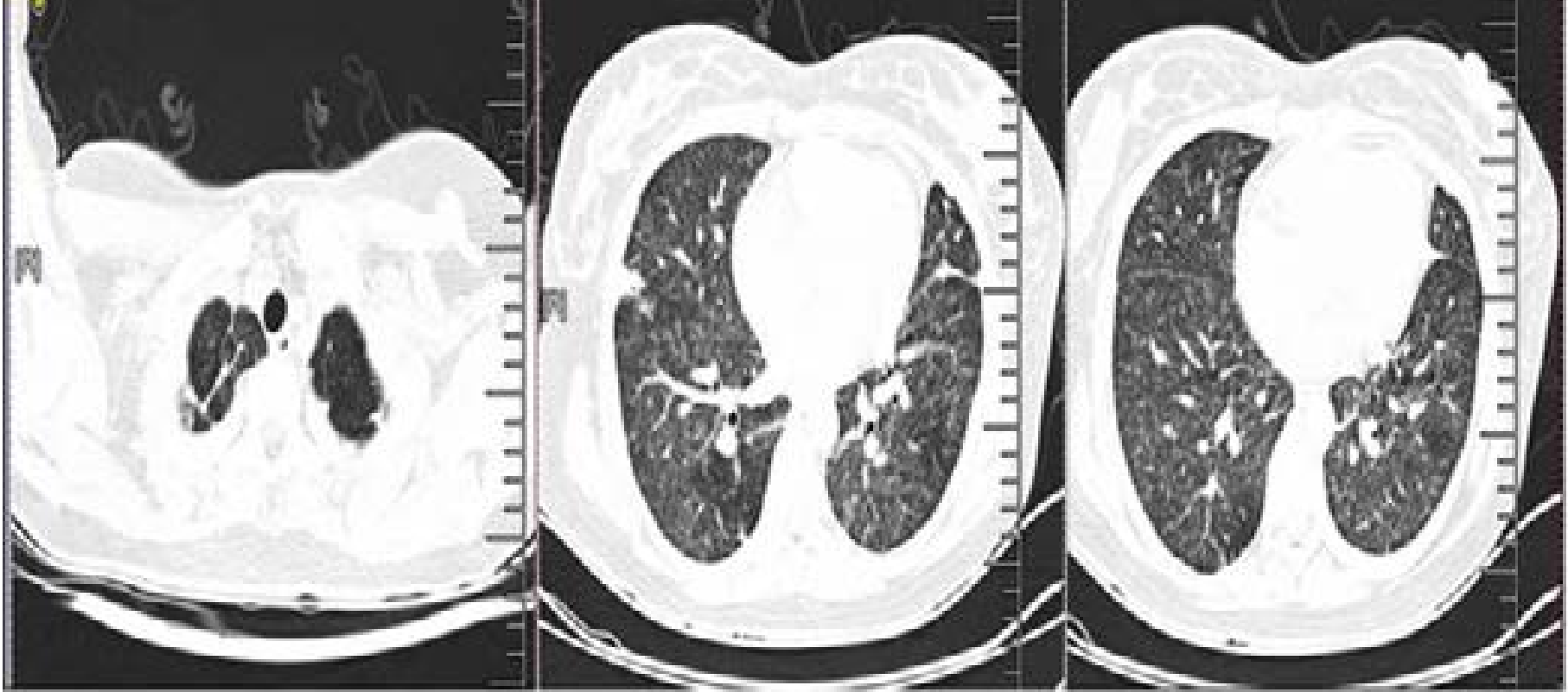

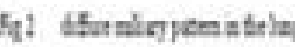

Discussion

It is well established that genital tuberculosis is one of the most common causes of female infertility ${ }^{2}$. In female genital tuberculosis patients, it affects the fallopian tubes and the endometrium and may cause infertility, the incidence of infertility in genital tuberculosis world wide varies from 44 to $74 \%{ }^{3}$. Involvement of the endometrium is observed in $50-60 \%$ of the genital tubeculosis patients ${ }^{4}$. In China we have no true prevalence. However, it is difficult to predict the accurate prevalence of genital tuberculosis because it often tends to exist without any clinical signs or symptoms.

Military tuberculosis is due to hematogenous spread of tubercle bacilli. It is often the consequence of primary infection in children, in adults it may be due to either recent infection or reactivation of old disseminated foci 5, when their immune function is lower, such as longtime using immunosuppressive agents (e.g., glucocorticoid, anti-cancer drugs, etc), immunocompromised persons (e.g., patients with HIV infection), combined with chronic disease or the elderly and so on. Military tuberculosis has showed relatively specific images, so most of the time a high index of suspicion is required for the images to diagnose military tuberculosis.

During pregnancy, the maternal immune system shows a relative bias toward $T$ helper type 2 immunity. These alterations lead to impaired cell-mediated immunity with increased susceptibility to certain infections such s tuberculosis ${ }^{6}$. In addition to the change of immune system during pregnancy, after IVF-ET the patient took dydrogesterone orally everyday to protect the fetus. Dydrogesterone is a kind of progesterone which affects the cellular immune function just like glucocorticoids? Our patient didn't have any signs and symptoms of tuberculosis and she had no his pulmonary infection. However, the first chest CT scan showed the hyperplasia and calcified lesions in the bilateral upper lobes of the lung. Actually she had had the Mycobacterium tuberculosis infection before, that may have been the reason for her bilateral tubal occlusion and the latent foci was reactive and disseminated when she got pregnant.

\section{Conclusion}

More than 5 million new cases of tuberculosis (all forms, both pulmonary and extrapulmonary) were reported to the World Health Organization (WHO) in 2005, >90\% of cases were reported from developing countries ${ }^{2}$. China is the most populous developing country in the world, so prevention and control of tuberculosis is very mportant. With the development of assisted reproduc tive technology, more and more patients with infertility get pregnant by IVF-ET. Thus, before IVF-ET operation, clinicians should exclude tuberculosis for the infertility patients especially with bilateraltubal occlusion order to avoid tuberculosis disseminate during pregnancy. 


\section{Follow up:}

The patient was transferred to the specialised hospital and started on four-drug chemotherapy with isoniazid, rifampin, pyrazinamide, and ethambutol. Unfortunately, after two weeks the patient had an abortion. One month later clinical symptoms had completely disappeared.

\section{References}

1. World Health Organization: Guidelines for the programmatic management of drug-resistant tuberculosis. Geneva; 2006.

2. Sutherland A. The changing pattern of tuberculosis of the female genital tract: a thirty year survey. Arch Gynecol Obstet 1983; 234:95-101.
3. Tripathy SN, Tripathy SN. Infertility and pregnancy outcome in female genital tuberculosis. Fertil Steril 2002; 76:159-163.

4. Gurgan T, Demirol A. Tuberculosis in assisted reproduction and infertility. Int Congr Ser 2004; 1266:287-94. 5. Harrison's PRINCIPLES OF INTERNAL MEDICINE. Seventeenth Edition. Chapter 158.

6. Yip L, MeCluskey J, Sinclair R. Immunological aspects of pregnancy. Clin Dermatol 2006; 24:84-87.

7. Xiangf Song, Xiang Sun, Hui Wang. Regulatory effect of estrogen and progesterone on the growth of $\mathrm{T}$ cells. Chinese Journal of Celluar and Molecular Immunology 2005; 21: 249-50. 